\title{
Performance analysis in esports: part 1 - the validity and reliability of match statistics and notational analysis in League of Legends
}

\author{
Andrew R. Novak,2, Kyle J. M. Bennett3,4, Matthew A. Pluss', and Job Fransen¹ \\ ${ }^{1}$ Human Performance Research Centre, Sport and Exercise Science, Faculty of \\ Health, University of Technology Sydney, Moore Park, Australia \\ ${ }^{2}$ High Performance Department, Rugby Australia, Moore Park, Australia \\ 3 School of Health and Human Sciences, Southern Cross University, Coffs Harbour, \\ Australia. \\ 4 Centre for Athlete Development, Experience \& Performance, Southern Cross \\ University, Coffs Harbour, Australia.
}

\begin{abstract}
Performance analysis in sports objectively captures aspects of athlete performances to inform coaching. Comparatively, esports is an emerging expertise domain with limited performance analysis research, however, no research has yet investigated the quality of available data. Therefore, this research aimed to: 1) assess the validity of publicly accessible Match History statistics from professional League of Legends matches; 2) assess the agreement of notational analysis between three experienced players; and 3) assess the agreement between a novice and Match Histories. 30 professional matches were randomly selected from the North American and European 2019 seasons. The Match Histories for each match were copied from the publicly accessible repository, while each of the authors independently viewed videos on the public Video On Demand repository, and encoded action variables corresponding to the Match Histories. Data were compared 1) between the most experienced player and Match Histories; 2) between the three experienced authors; and 3) between the novice author and Match Histories. Krippendorff's Alpha was calculated with acceptable agreement set at $\alpha \geq 0.8$. The most experienced player was in good agreement with Match Histories ( $\alpha=0.868-1.000)$, while the novice rater was in good agreement except for First Tower Time $(\alpha=0.764)$ and First Inhibitor Team (0.740). The three experienced players were in good agreement $(\alpha=0.861-$ 1.000). Match Histories and experienced player annotations can be used interchangeably for all observed measures to facilitate performance analysis in professional League of Legends. A novice can be used to capture some basic statistics, while other measures require domain expertise.
\end{abstract}

\section{Key Words}

Esports, Performance, Notational Analysis, Video Games, Technology.

\section{DOI}

http://dx.doi.org/10.31236/osf.io/sm3nj

\section{Citation}

Novak, A. R., Bennett, K. J. M., Pluss, M. A., \& Fransen, J. (2019). Performance analysis in esports: part 1 - the validity and reliability of match statistics and notational analysis in League of Legends. SportRxiv. doi:10.31236/osf.io/sm3nj 


\section{INTRODUCTION}

\subsection{Performance analysis}

Performance analysis in sport encompasses methodologies that measure aspects of athlete performances to provide feedback and inform coaching (Hughes \& Franks, 2004). A common method of performance analysis is notational analysis, whereby domain experts such as coaches or performance analysts view video footage or live performances and encode data files to describe the timing, location and frequencies of action variables such as tackles, passes, kicks, penalties and goals (Bennett, Bezodis, Shearer, Locke \& Kilduff, 2019; Hughes \& Bartlett, 2002; Hughes \& Franks, 2004; Parmar, James, Hughes, Jones \& Hearne, 2017). This process ultimately leads to the development of Performance Indicators, which have been defined as a "selection, or combination, of action variables that aims to define some or all aspects of a performance” (Hughes \& Bartlett, 2002; Hughes, 2004). Performance Indicators can then be analysed and developed into performance profiles to describe various aspects of performance as part of a feedback system to the coach and athlete (Hughes, Evans \& Wells, 2001; Lames \& McGarry, 2007). Understanding the relationships between actions and performance outcomes helps coaches and players to develop effective training strategies and can guide further explorations into the underlying mechanisms (Hughes, Barnes, Churchill \& Stone, 2017; McGarry, 2009; Ortega, Villarejo, \& Palao, 2009).

Within the sports domain, performance analysis is typically conducted either by coaches or performance analysts situated within an individual team or organisation, or by external commercial organisations. These external organisations capture data for entire competitions, and teams may then purchase access licenses to their own data and/or that of the competition. Importantly, Hughes \& Franks (2004) have raised concerns over the validity of notational analysis systems, highlighting the potential for software and hardware to introduce errors, and for user input errors to occur. Given these concerns, inter-rater 
reliability is often assessed with respect to internal processes (Hughes et al., 2017; James, Mellalieu \& Jones, 2005) as well as external notational analysis providers (Bishop \& Barnes, 2013; Robertson, Gupta \& McIntosh, 2016; Vaz, Rooyen \& Sampaio, 2010) to facilitate rigorous performance analysis and research. Collectively, coaches and analysts within sports have developed methodologies to facilitate and validate performance analysis. Comparatively, electronic sports (esports) has recently emerged as a new expertise domain in which research concerning performance analysis is limited. Therefore, esports coaches and analysts may benefit from the methodologies established in sports, also allowing sports practitioners to apply their expertise in a new domain.

\subsection{Esports}

Electronic sports (esports) is a modern competitive environment where human players compete in video game competitions via human-computer interactions (Pluss et al., 2019), thus, differentiating esports from the broader field of video gaming. The rapid growth in esports popularity has led to the domain becoming more professional, with many players contracted on a full-time basis and competing in both national and international competitions. Further, esports competitions are now posting staggering prize pools across several disciplines e.g. Dota 2 - the International 2019 (\$34 million USD), Fortnite - World Cup Finals 2019 (\$15 million USD), and League of Legends - World Championship 2018 (\$6 million USD) (retrieved 24/09/2019 from https://www.esportsearnings.com/tournaments). Given this growth, it is unsurprising that researchers have begun to explore esports and video gaming. However, typically these have explored the relationships between video gaming and esports vs. domain general factors such as working memory and information processing ability (Bonny \& Castaneda, 2017, Colzato, van den Wildenberg, Zmigrod, \& Hommel, 2013; Powers, Brooks, Aldrich, Palladino \& Alfieri, 2013), while no research has yet investigated the match-related factors that underpin successful performance within esports competitions (i.e. performance 
indicators). As such, coaches and players must rely on information that is considered poor scientific quality (e.g. anecdotal observations, individual experiences, and unvalidated statistics). Anecdotal reports reveal that professional Oceanic esports coaches and players rely on knowledge derived from other disciplines that are considered somewhat similar such as chess and sports (personal communication, [April 2019]), while some researchers have also drawn links between esports and chess (Bonny \& Castaneda, 2016; Pluss et al., 2019). As a result, research on performance indicators in esports is required to provide esports coaches and players with a greater quality of domain-specific evidence that can inform practice. Esports games providers offer a plethora of match data (often made publicly available on websites or via application program interfaces), which are facilitated by the inherent softwarebased nature of the games and are automatically captured throughout a match. However, unlike sports, no research has investigated the validity or reliability of these automated match statistics and conducting performance analysis using such data would be questionable. Therefore, this study aimed to investigate the validity and reliability of match statistics and notational analysis in esports, specifically focusing on League of Legends as it is a game which three of the four authors are experienced as players and consultants. It is anticipated that this research will serve as an exemplar to validate the collection of match statistics in esports games, which is a necessary precursor to undertaking performance analysis in this new domain.

\subsection{League of Legends}

League of Legends is an esports discipline of the Multiplayer Online Battle Arena genre. It is one of several globally popular esports games (ranging from between 200,000 ranked players in regions such as Oceania and Russia, to more than 3.5 million ranked players in Korea (data extracted via website https://[region].op.gg/statistics/tier, 15/o9/2019)). Two teams of five players work to achieve a primary objective - destroy the opposing team's Nexus 
Performance analysis in esports: part 1 - the validity and reliability of match statistics and notational analysis in League of Legends

(the main structure within their home base) located at the opposite side of the arena (Figure 1). At the start of a match, each team is designated to either the blue side (bottom left) or red side (top right). The arena is divided into three lanes (top, middle, and bottom), with a jungle area between each lane. Visually, a river passes from the top left to bottom right, separating the two territories of each team. The match continues until one team destroys the other team's Nexus i.e. draws are not possible. Players, also known as Summoners, select from a pool of 145 unique Champions (the current champion pool size as of September, 2019) and control their selected Champion throughout the match. The Champions are avatars controlled by the players, and each has unique attributes and skills that can be performed. Players move their Champion through the arena any way they wish, although the most common strategy is to allocate one player to the top lane, one player to the mid lane, two players to the bottom lane, and one player to the jungle. Spreading the five teammates across the arena in this manner is not a requirement and has been subject to change in rare circumstances, but has evolved as the most common practice.

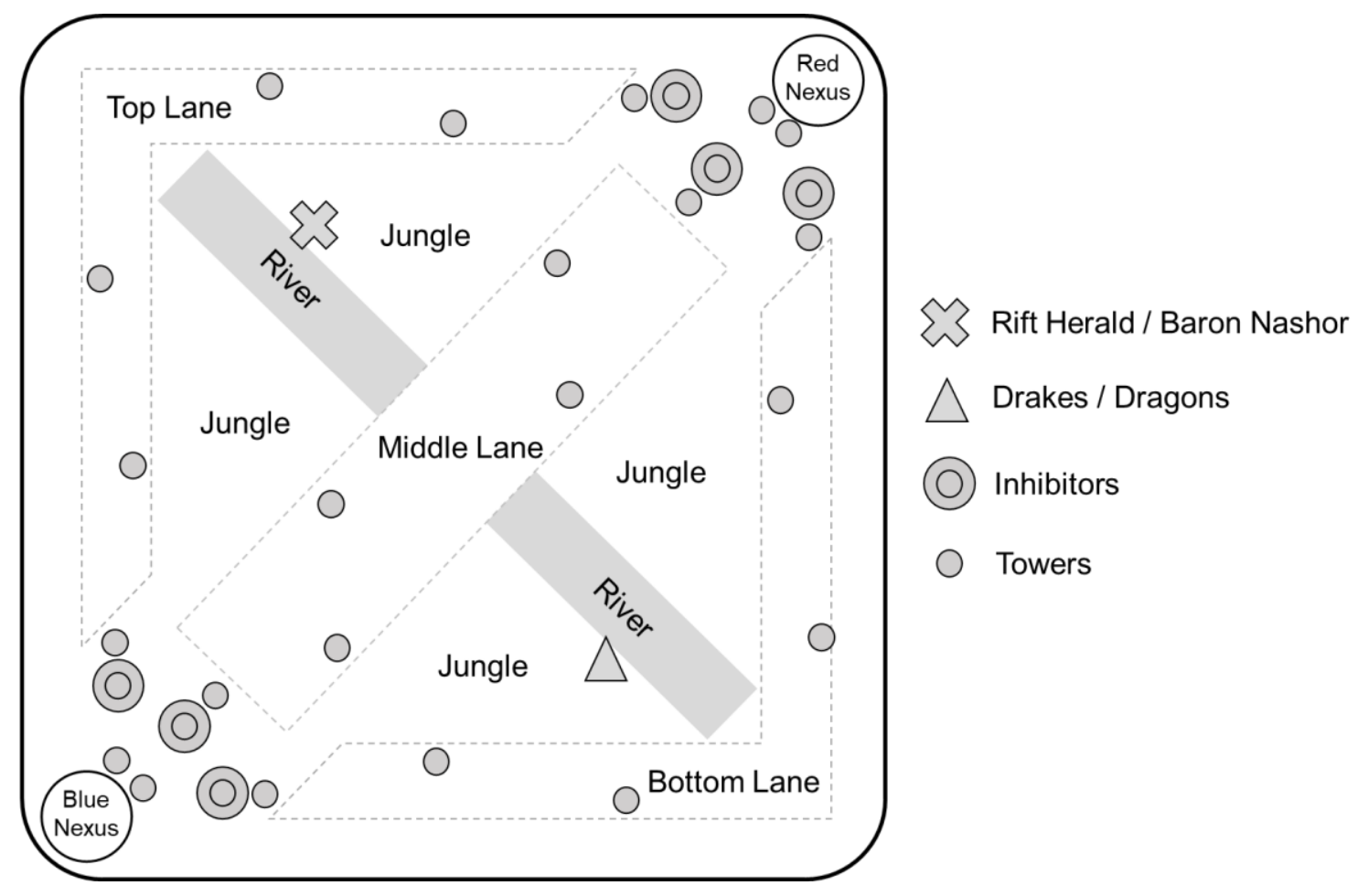

Figure 1: League of Legends arena 
During a match, players face various enemies and objectives including: the five opposing team players, waves of computer-controlled Minions, Jungle Monsters, enemy buildings called Towers, and larger jungle Monsters that only spawn at specific times such as Elemental Drakes, the Elder Dragon, Rift Herald, and Baron Nashor. Attacking each of these enemies and objectives awards experience (used to "level-up" the players' champions), and/or gold (in-game currency used to purchase items), each of which are used to make Champions more powerful via awarding new abilities or increasing the five primary attributes of attack damage, ability power, armour, magic resistance, and health. In addition to simply attacking targets and objectives, bonus rewards are provided for acquiring key objectives such as First Blood (the player(s) to obtain the first kill in a match is awarded extra gold) First Tower (the player(s) to destroy the first tower is awarded extra gold), the Elder Dragon (increases the effects applied by slaying previous Elemental Drakes), the Rift Herald (the player is provide an additional powerful ability to be used at a later time) and Baron Nashor (provides all surviving team mates with temporary bonus damage and an aura to amplify the power of their team's nearby Minions). As champions become more powerful, they can overcome objectives more easily that can ultimately aid in winning the match.

Given the lack of research regarding performance indicators and notational analysis in esports, this study was developed with three aims: first, to investigate the validity of professional League of Legends match statistics when compared to notational analysis by an experienced player; second, to determine the inter-rater reliability of notational analysis from League of Legends video footage by three experienced players; and third, to determine whether a novice with minimal training could be utilised to capture basic match statistics in League of Legends. It was hypothesised: 1) that the Match Histories would be valid (i.e. in agreement) with the experienced player's video annotations; 2) that the three experienced players' video annotations would be in agreement; and 3) that the novice's annotations would be in agreement with the Match Histories. 
Performance analysis in esports: part 1 - the validity and reliability of match statistics and notational analysis in League of Legends

\section{MATERIALS AND METHODS}

\subsection{Sample}

Data for this study were collected from 30 professional League of Legends matches, which were obtained via publicly accessible repositories of Match History statistics and video footage. Specifically, on the $28^{\text {th }}$ of July 2019 , the 2019 Summer Season match schedules were accessed from the North American League of Legends Championship Series (NA LCS) and the League of Legends European Championship (EU LEC) (https://watch.lolesports.com/schedule?leagues=lcs,lec). These two competitions were selected because they each contain a statistics view alongside the video footage, presenting live data which is not always visible within the video footage (e.g. the quantity of Gold and number of Wards for individual players) and was not available for other competitions at the time of data collection. Each match from this season to date (NA LCS: $n=70$; EU LEC: $n=60$ ) were placed into Microsoft Excel spreadsheets, with each match allocated to individual rows in sequential order (by date and time). A random function ("Randbetween”) was applied 15 times to each competition to identify 15 matches for analysis, combining for a total of 30 matches. Institutional ethics approval was received prior to undertaking this study.

The official match histories were located via the public League of Legends repository (https://matchhistory.na.leagueoflegends.com) while video footage was located via the public League of Legends Video On Demand (VOD) repository (https://watch.lolesports.com/vods) Match histories and video footage were initially accessed and assessed by the lead author to ensure that all data were populating as expected. During this process, it was noted that the statistics view did not populate with data alongside four of the identified videos, while the match histories did not populate with data for two of the identified matches. In each of these cases $(n=6)$, the matches were removed from the study and new matches were identified 
using the randomised function previously described. There were no cases identified in which neither the video footage nor the match histories populated.

\subsection{Procedure}

The match statistics identified for validation were statistics that were available in both the Match Histories and video footage. Variables included three categories: 1) frequency data (e.g. number of kills, deaths and assists for each player); 2) time-dependent data (e.g. the exact time that the first tower was destroyed); and 3) categorical data (e.g. which team destroyed the first tower). Table 1 display the complete list of variables. The lead author developed a spreadsheet that allowed each of the co-authors to independently access video footage for the 30 matches, and to record the identified match statistics from each match, without the possibility of observing other authors' records. The match statistics were copied from the Match History pages into a spreadsheet by the lead author.

Three of the authors (AN, KB, and MP) each had experience (255, 1596 and 1401 hours, respectively) playing League of Legends (data extracted from user accounts via website: https://wol.gg/ data extracted 03/08/2019), and had also consulted with a professional League of Legends team for two years. However, one of the authors (JF) had no prior experience playing or viewing League of Legends. Therefore, a one-hour training session was administered by the lead author, allowing JF to subsequently identify and record the variables from match videos. Agreement was then determined between JF and the Match Histories to determine whether a novice rater could be utilised to capture League of Legends match statistics from video footage. 
Performance analysis in esports: part 1 - the validity and reliability of match statistics and notational analysis in League of Legends

\subsection{Statistical analysis}

Data were formed into three datasets using Microsoft Excel spreadsheets. Dataset 1 consisted of the most experienced player's $(\mathrm{KB})$ measures obtained from video footage and the Match Histories. Dataset 2 consisted of measures obtained by each of the three experienced authors from video footage (AN, KB, and MP). Dataset 3 consisted of the novice rater's (JF) measures obtained from video footage and the Match Histories. Inter-rater reliability was calculated for each of the three data sets via Krippendorff's alpha (Krippendorff, 1970). This statistic was deemed appropriate as it can be calculated across any number of raters, and for multiple data types (Hayes \& Krippendorff, 2007), resulting in consistent interpretations of agreement across each of the three analyses. Analyses were conducted via the IRR package (vo.84.1, Gamer, Lemon, Fellows \& Singh, 2019) within R, using the "kripp.alpha” function and setting the appropriate method (e.g. ordinal or nominal). Time-series variables were converted to integers (e.g. $27 \mathrm{~min} 40 \mathrm{~s}=1660$ ), facilitating analysis of these variables as ordinal data. Krippendorff's alpha values were interpreted as acceptable where $\alpha \geq 0.8$ according to prior recommendations (Krippendorff, 2004, p. 241). For the analyses of Datasets 1 and 3 where the most experienced author and novice author were each compared separately with the Match Histories, an intra-class correlation coefficient (ICC) and root mean square error (RMSE) were also calculated to facilitate interpretation of the errors and to enable comparisons to be drawn with prior research within sports.

\section{RESULTS}

The summary match statistics from Match History data are presented in Table 1 to facilitate the contextual interpretation of agreement and RMSE values. Agreement between each of the three experienced raters is presented in Table 2. Agreement was acceptable for all measures $(\alpha$ range $=0.861-1.000$ ), with three variables exhibiting perfect agreement. The perfect agreements were for Match Outcome, Rift Herald Team, and Elemental Drakes, while 
Performance analysis in esports: part 1 - the validity and reliability of match statistics and notational analysis in League of Legends

the lowest agreement was for First Inhibitor Team $(\alpha=0.861)$ and number of Barons $(\alpha=$ 0.866).

Table 1: Match statistics for North American and European professional League of Legends competition

\begin{tabular}{|c|c|c|c|c|}
\hline & \multicolumn{2}{|c|}{ North America $(n=15)$} & \multicolumn{2}{|c|}{ Europe $(n=15)$} \\
\hline & Blue Side & Red Side & Blue Side & Red Side \\
\hline Win Rate (\%) & 67 & 33 & 53 & 47 \\
\hline Match Duration (mm:ss) & \multicolumn{2}{|c|}{$32: 41 \pm 04: 59$} & \multicolumn{2}{|c|}{$32: 07 \pm 06: 50$} \\
\hline Level & $75.6 \pm 6.6$ & $73.7 \pm 6.8$ & $75.1 \pm 8.5$ & $73.1 \pm 9.9$ \\
\hline Gold (in thousands) & $59.1 \pm 9.2$ & $56.3 \pm 9.9$ & $59.8 \pm 11.7$ & $57 \cdot 5 \pm 15 \cdot 4$ \\
\hline Creep Score & $1076.3 \pm 158.8$ & $1056.7 \pm 175.6$ & $1074.0 \pm 240.3$ & $1036.8 \pm 244.9$ \\
\hline Kills & $12.0 \pm 4.1$ & $8.6 \pm 5.9$ & $14.6 \pm 5 \cdot 7$ & $12.2 \pm 7.4$ \\
\hline Deaths & $8.6 \pm 5.9$ & $12 \pm 4.1$ & $12.2 \pm 7.4$ & $14.6 \pm 5 \cdot 7$ \\
\hline Assists & $29.6 \pm 11.4$ & $20.9 \pm 14.9$ & $32.1 \pm 15.0$ & $26.9 \pm 17.4$ \\
\hline Wards Placed & $119.3 \pm 29.2$ & $118.0 \pm 27.4$ & $105.4 \pm 26.9$ & $113.0 \pm 39.0$ \\
\hline Wards Destroyed & $55 \cdot 7 \pm 15 \cdot 3$ & $49.9 \pm 19.4$ & $48.9 \pm 18.8$ & $42.1 \pm 17.8$ \\
\hline Towers Taken (Out of 11) & $7 \cdot 5 \pm 3.5$ & $5.2 \pm 3.6$ & $6.7 \pm 3.7$ & $5.6 \pm 4.2$ \\
\hline $\begin{array}{l}\text { Inhibitors Taken (Out of } \\
\text { 3) }\end{array}$ & $1.6 \pm 1.5$ & $0.73 \pm 1.0$ & $1.1 \pm 1.1$ & $0.9 \pm 1.1$ \\
\hline Dragons & $2.7 \pm 1.3$ & $1.6 \pm 1.2$ & $2.0 \pm 1.1$ & $1.9 \pm 1.3$ \\
\hline First Bloods (\%) & 33 & 67 & 73 & 27 \\
\hline First Blood Time (mm:ss) & \multicolumn{2}{|c|}{$05: 25 \pm 01: 47$} & \multicolumn{2}{|c|}{$05: 39 \pm 02: 32$} \\
\hline First Towers (\%) & 53 & 47 & 53 & 47 \\
\hline First Tower Time (mm:ss) & \multicolumn{2}{|c|}{$14: 09 \pm 02: 48$} & \multicolumn{2}{|c|}{$14: 26 \pm 01: 35$} \\
\hline Heralds (\%) & 47 & 53 & 53 & 47 \\
\hline First Barons (\%) & 53 & 47 & 40 & 53 \\
\hline Barons & $0.67 \pm 0.49$ & $0.73 \pm 0.70$ & $0.53 \pm 0.51$ & $0.67 \pm 0.72$ \\
\hline First Inhibitors (\%) & 67 & 33 & 53 & 47 \\
\hline First Elder Dragons (\%) & 7 & 7 & $\mathrm{o}$ & 13 \\
\hline Elder Dragons & $0.07 \pm 0.26$ & $0.07 \pm 0.26$ & o & $0.13 \pm 0.35$ \\
\hline
\end{tabular}

Note: Creep Score is a combination of Minion and Monster kills. 
Performance analysis in esports: part 1 - the validity and reliability of match statistics and notational analysis in League of Legends

Table 2: Agreement between three raters with domain experience

\begin{tabular}{lc}
\hline & $\alpha$ \\
\hline Match Duration & 0.999 \\
First Blood Time & 0.998 \\
First Tower Time & 0.993 \\
Match Outcome & 1.000 \\
First Blood Team & 0.911 \\
First Tower Team & 0.955 \\
Rift Herald Team & 1.000 \\
First Baron Team & 0.962 \\
First Inhibitor Team & 0.861 \\
First Elder Dragon Team & 0.899 \\
Level & 0.997 \\
Gold & 0.995 \\
Creep Score & 0.998 \\
Kills & 0.992 \\
Deaths & 0.992 \\
Assists & 0.987 \\
Wards Placed & 0.997 \\
Wards Destroyed & 0.992 \\
Towers & 0.937 \\
Inhibitors & 0.975 \\
Elemental Drakes & 1.000 \\
Barons & 0.866 \\
Elder Dragons & 0.903
\end{tabular}

Note: $\alpha=$ Krippendorff's Alpha; Creep Score is a combination of Minion and Monster kills.

For the inter-rater reliability between the most experienced player and the Match Histories (Table 3), agreement was acceptable for all measures ( $\alpha$ range $=0.868-1.000$, ICC $=0.866-1.000, \mathrm{RMSE}=0.000-26.311)$, with 13 variables exhibiting perfect agreement For the inter-rater reliability between the novice rater and the match history data (Table 3), agreement was acceptable for all except two measures. Specifically, agreement was only 0.740 for determining the team to obtain first inhibitor and 0.764 for determining the time that the first tower was taken. Remaining variables were in the acceptable range ( $\alpha$ range $=0.855-$ 
1.000, ICC $=0.866-1.000$, RMSE $=0.000-17.962$ ), with 11 variables exhibiting perfect agreement.

Table 3: Agreement between the most experienced author and Match History; and a novice rater with no domain experience and Match History

\begin{tabular}{lcccccc}
\hline & \multicolumn{3}{c}{ Experienced rater } & \multicolumn{3}{c}{ Novice rater } \\
& $\alpha$ & ICC & RMSE & $\alpha$ & ICC & RMSE \\
\hline Match Duration & 0.995 & 1.000 & 5.027 & 0.995 & 1.000 & 5.604 \\
First Blood Time & 0.998 & 0.999 & 4.590 & 1.000 & 1.000 & 0.707 \\
First Tower Time & 0.990 & 0.996 & 12.412 & 0.764 & 0.783 & 7.819 \\
Match Outcome & 1.000 & 1.000 & 0.000 & 0.931 & 0.932 & 0.183 \\
First Blood Team & 0.868 & 0.866 & 0.258 & 1.000 & 1.000 & 0.000 \\
First Tower Team & 0.934 & 0.935 & 0.183 & 0.868 & 0.866 & 0.258 \\
Rift Herald Team & 1.000 & 1.000 & 0.000 & 0.873 & 0.916 & 0.408 \\
First Baron Team & 1.000 & 1.000 & 0.000 & 1.000 & 1.000 & 0.000 \\
First Inhibitor Team & 1.000 & 1.000 & 0.000 & 0.740 & 0.779 & 0.658 \\
First Elder Dragon Team & 1.000 & 1.000 & 0.000 & 1.000 & 1.000 & 0.000 \\
Level & 0.998 & 0.999 & 0.408 & 0.998 & 0.999 & 0.408 \\
Gold & 1.000 & 1.000 & 0.177 & 0.993 & 0.997 & 0.992 \\
Creep Score & 0.999 & 0.992 & 26.311 & 1.000 & 1.000 & 1.342 \\
Kills & 1.000 & 1.000 & 0.000 & 1.000 & 1.000 & 0.183 \\
Deaths & 1.000 & 1.000 & 0.000 & 1.000 & 1.000 & 0.183 \\
Assists & 1.000 & 1.000 & 0.000 & 1.000 & 0.999 & 0.516 \\
Wards Placed & 0.863 & 0.906 & 17.595 & 0.855 & 0.902 & 17.962 \\
Wards Destroyed & 0.883 & 0.913 & 9.670 & 0.873 & 0.911 & 9.878 \\
Towers & 0.997 & 0.999 & 0.129 & 0.998 & 0.995 & 0.387 \\
Inhibitors & 0.995 & 0.994 & 0.129 & 0.989 & 0.994 & 0.129 \\
Elemental Drakes & 1.000 & 1.000 & 0.000 & 0.996 & 0.995 & 0.129 \\
Barons & 0.934 & 0.933 & 0.224 & 0.973 & 0.977 & 0.129 \\
Elder Dragons & 1.000 & 1.000 & 0.000 & 1.000 & 1.000 & 0.000 \\
\hline
\end{tabular}

\section{DISCUSSION}

The current study is the first to investigate the validity and reliability of match statistics from professional League of Legends competitions. This process is essential to determine whether these data can be used to develop future performance analysis studies in esports, and whether data obtained from Match Histories conform to those obtained from experienced and 
novice performance analysts observing and annotating games. More specifically, this study used three analyses to determine: 1) whether publicly accessible Match Historers were valid with respect to an experienced player's annotations; 2) whether three experienced League of Legends players were in agreement when annotating data from video footage; and 3) whether a novice's annotations were in agreement with Match Histories. It was found that the agreement between the most experienced player and Match Histories, and between the three experienced players were acceptable ( $\alpha \geq 0.8)$ across all measures. Comparatively, the novice's ratings fell below the acceptable threshold for First Tower Time and First Inhibitor Team.

The level of agreement observed within this study is similar to previous research in sports such as Australian Football and Rugby, in which inter-rater reliability was calculated for match annotations (Hughes et al., 2017; Robertson et al., 2016; Vaz et al., 2010). Robertson et al (2016) compared the lead author's observations with a competition-wide external match statistic provider and observed ICC and RMSE within the ranges of $0.947-1.000$ and $0-4.5$, respectively, resulting in "very high agreement". Hughes et al. (2017) and Vaz et al. (2010) each compared the ratings of two experienced analysts and observed kappa values of $\geq 0.91$ and o.88-0.98, respectively, with Hughes and colleagues qualifying their observed kappa values as “a very good level of agreement", while Vaz and colleagues did not provide a qualifier for their observed kappa values. Ultimately, these findings reflect that analysts can accurately identify and record action variables which can be contextualised and developed into performance profiles, thereby facilitating a performance feedback mechanism.

In comparison to the aforementioned sports research which utilised only 2-10 matches, this study analysed 30 matches across two professional competitions. Furthermore, almost all variables observed in this study were in very good agreement based on the accepted assessments of agreement in the aforementioned research. However, while the agreement 
between the most experienced player and the Match Histories were relatively low for Wards Placed ( $\alpha=0.863)$ and Wards Destroyed $(\alpha=0.883)$, the agreement between the three experienced players for these same measures was near-perfect $(\alpha=0.997$ and 0.992, respectively). This indicates variance between video footage and the Match Histories for these variables. While the alpha value is within the acceptable range, it is noted that the acceptable range is assigned somewhat arbitrarily, and agreement should be interpreted within its context. Specifically, the variance between experienced player annotation and Match Histories translates to a RMSE of 17.595 and 9.670 for Wards Placed and Wards Destroyed, respectively. This is larger than the range (0-4.5) observed by Robertson et al. (2016). However, it should be noted that the RMSE is specific to the units and magnitude of the individual measure. Therefore, while the RMSE for Wards Placed was 1.8 times greater than the RMSE for Wards Destroyed, the magnitude of Wards Placed (e.g. 119.3 \pm 29.2 for North America Blue Side) was also 2.1 times greater than Wards Destroyed (e.g. $55 \cdot 7 \pm 15 \cdot 3$ for North America Blue Side). Further investigation revealed that there were no cases in which Wards Placed or Wards Destroyed were recorded as being greater in the videos than what was reflected in the Match Histories. Given the relatively high errors in these variables, it is unlikely that they can be used for performance analysis, however, further analysis with respect to match outcomes is required to determine what the acceptable levels of error may be.

While good agreement was expected for both the experienced player and the novice given the relative simplicity of the annotations (no subjective assessments required), it was interesting to observe that the novice achieved a smaller RMSE than the experienced rater for First Tower Time (7.819 vs 12.412) and Creep Score (26.311 vs 11.342). This observation prompted further exploration, and it was found that the high RMSE for these two measures recorded by the experienced player could be attributed to one outlier for each variable. Specifically, in one match, the experienced player recorded a First Tower Time which was 59 s greater than the Match History, while in another match, the Creep Score was recorded to be 
202 less than the Match History. These errors were assumed to be user input errors. To test this theory, the links to those two videos were emailed to the experienced player with instructions to record the First Tower Time from Video 1, and the Creep Score from Video 2. As expected, the responses to these second observations were more aligned with the Match Histories (errors reduced from 59 s and 202 Creep Score to o s and o Creep Score i.e. perfect agreement in both cases), indicating that user input error had cause the high RMSE for these two measures in the experienced player's initial annotations. This process has supported the concerns raised by Hughes \& Franks (2004) regarding errors in notational analysis, and great care is required when manually entering data from any source.

It was interesting to observe that the novice rater achieved perfect or near-perfect agreement for many variables. This is likely due to the fact that the video annotation for League of Legends was largely facilitated by recording data that are visible on-screen at various moments of the match (e.g. the number of kills, deaths and assists are visible almost continuously throughout the match and can therefore be easily recorded). For such variables, the only expected error would be due to manual input errors as we noted above. This differs from sports annotation, in which there are no numbers directly perceivable from the performance environment, but rather each individual event must be somewhat subjectively rated prior to notation. In these cases, there are three opportunities for error: 1) errors in perception (i.e. seeing the event), 2) errors in subjective assessment of the event (e.g. subjectively coding a long vs. a medium-ranged kick); 3) manual input error. The two variables that fell below the acceptable thresholds of agreement were the team to obtain First Inhibitor, and the time that the First Tower was taken. Given that the annotations in this study were conducted retrospectively rather than live, these two variables required the authors to filter through the video footage to find when the events occurred and to record the appropriate team and timestamp respectively. The results support that a novice rater is not suitable to record such variables and a greater level of domain expertise is required. Separately, the lowest 
agreement between the three experienced players were for First Inhibitor team $(\alpha=0.861)$ and number of Barons (o.866). It should be noted that each of these two objectives respawns after 5 minutes and 7 minutes respectively, making them more difficult to locate retrospectively when viewing video footage. We suggest that greater levels of domain expertise and care are required to accurately capture such variables.

\section{LIMITATIONS}

This study should be taken with limitations in mind. In contrast to sports annotation, the observers in this study did not manually annotate each individual event but rather recorded the numbers available on-screen at relevant moments of the game. However, the authors have collectively obtained $>3000$ hours game experience, have observed hundreds of hours of professional competitions, consulted with a professional League of Legends team for two years, and personal communications prior to and during this study (July-September 2019) revealed that no situations were recalled in which the data on-screen did not reflect the actual game state. Furthermore, this study assessed validity of Match Histories with respect to an experienced player's video annotations, as it is not possible to validate the data at a programmatic level using a true criterion given the proprietary software-based nature of the domain. Through a combination of our domain experience and the various analyses in this study, we are confident that collecting data from Match Histories and video annotation in this manner is a valid method of notational analysis in League of Legends.

\section{CONCLUSION}

This study demonstrates that numerous variables can be validly and reliably collected from professional League of Legends competitions, with strong agreement between Match Histories and notational analysis. Some variables were noted to require domain experience and were 
not suitable for collection by a novice analyst (First Tower Time and First Inhibitor Team), while other variables such as the number of Wards Placed and Wards Destroyed were not consistent between Match Histories and experienced player annotations. In all other cases, Match Histories and video annotations could be reliably interchanged e.g. when Match Histories do not populate with data as noted for several matches included within this study. However, it is not yet known which variables could be considered as key performance indicators. Further research is required to determine the most appropriate methods for contextualising action variables as performance indicators, and to determine which performance indicators are most relevant to performance outcomes in League of Legends competition. These processes will ultimately enable researchers and practitioners to objectively measure and monitor player and team performances and inform strategical approaches to the game. The methodology adopted within this study can be extended to other esports domains to facilitate valid performance analysis.

\section{REFERENCES}

Bonny, J. W., \& Castaneda, L. M. (2017). Impact of the arrangement of game information on recall performance of Multiplayer Online Battle Arena players. Applied Cognitive Psychology 30(5): 664-671. DOI: 10.1002/acp.3234

Bonny, J. W., \& Castaneda, L. M. (2017). Number processing ability is connected to longitudinal changes in multiplayer online battle arena skill. Computers in Human Behavior 66: 377-387. DOI: 10.1016/j.chb.2016.10.005

Colzato, L., van den Wildenberg, W., Zmigrod, S. \& Hommel, B. (2013). Action video gaming and cognitive control: playing first person shooter games is associated with improvement in working memory but not action inhibition. Psychological Research 77(2): 234-239. DOI: 10.1007/s00426-012-0415-2

Bennett, M., Bezodis, D., Shearer, D. A., Locke, D., \& Kilduff, L. P. (2019). Descriptive conversion of performance indicators in rugby union. Journal of Science and Medicine in Sport 22(3): 330-334. DIO: 10.1016/j.jsams.2018.08.008

Bishop, L., \& Barnes, A. (2013). Performance indicators that discriminate winning and losing in the knockout stages of the 2011 Rugby World Cup. International Journal of Performance Analysis in Sport 13(1): 149-159. DOI: 10.1080/24748668.2013.11868638 
Hayes, A., \& Krippendorff, K. (2007). Answering the call for a standard reliability measure for coding data. Communication Methods and Measures 1(1): 77-89. DOI: 10.1080/19312450709336664

Hughes, M. (2004). Notational analysis - a mathematical perspective. International Journal of Performance Analysis in Sport 4(2): 97-139. DOI: 10.1080/24748668.2004.11868308

Hughes, A., Barnes, S., Churchill, S. M., \& Stone, J. A. (2017). Performance indicators that discriminate winning and losing in elite men's and women's Rugby Union. International Journal of Performance Analysis in Sport 17(4): 534-544. DOI: 10.1080/24748668.2017.1366759

Hughes, M. D., \& Bartlett, R. M. (2002). The use of performance indicators in performance analysis. Journal of Sports Sciences 20(10): 739-754. DOI: 10.1080/026404102320675602

Hughes, M., Evans, S., \& Wells, J. (2001). Establishing normative profiles in performance analysis. International Journal of Performance Analysis in Sport 1(1): 1-26. DOI: 10.1080/24748668.2001.11868245

Hughes, M \& Franks, I. (2004). Notational Analysis of Sport (2 ${ }^{\text {nd }}$ ed.). London, England: Routledge.

James, N., Mellalieu, S., \& Jones, N. (2005). The development of position-specific performance indicators in professional rugby union. Journal of Sports Sciences 23(1): 6372. DOI: 10.1080/02640410410001730106

Krippendorff, K. (1970). Estimating the reliability, systematic error and random error of interval data. Educational and Psychological Measurement 3o(1): 61-70. DOI: 10.1177/001316447003000105

Krippendorff, K. (2004). Content analysis: an introduction to its methodology ( $2^{\text {nd }}$ ed.). Thousand Oaks, California: Sage Publications, Inc.

Lames, M., \& McGarry, T. (2007). On the search for reliable performance indicators in game sports. International Journal of Performance Analysis in Sport 7(1): 62-79. DOI: 10.1080/24748668.2007.11868388

McGarry, T. (2009). Applied and theoretical perspectives of performance analysis in sport: Scientific issues and challenges. International Journal of Performance Analysis in Sport 9(1): 128-140. DOI: 10.1080/24748668.2009.11868469.

Ortega, E., Villarejo, D., \& Palao, J. M. (2009). Differences in game statistics between winning and losing rugby teams in the Six Nations tournament. Journal of Sports Science and Medicine 8(4): 523-527.

Parmar, N., James, N., Hughes, M., Jones, H., \& Hearne, G. (2017). Team performance indicators that predict match outcome and points difference in professional rugby league. International Journal of Performance Analysis in Sport. 17(6):1044-1056. Doi: 10.1080/24748668.2017.1419409 
Pluss, M. A., Bennett, K. J. M., Novak, A. R., Panchuk, D., Coutts, A. J., \& Fransen, J. (2019). Esports: the chess of the $21^{\text {st }}$ century. Frontiers In Psychology. 1O(156):1-5. Doi: 10.3389/fpsyg.2019.00156

Powers, K., Brooks, P., Aldrich, N., Palladino, M. A., \& Alfieri, L. (2013). Effects of video-game play on information processing: A meta-analytic investigation. Pychonomic Bulletin \& Review 2O(6): 1055-1079. DOI: 10.3758/s13423-013-0418-z

Robertson, S., Gupta, R., \& McIntosh, S. (2016). A method to assess the influence of individual player performance distribution on match outcome in team sports. Journal of Sports Sciences 34(19): 1893-1900. DOI: 10.1080/02640414.2016.1142106

Vaz, L., Rooyen, M., \& Sampaio, J. (2010). Rugby game-related statistics that discriminate between winning and losing teams in IRB and Super Twelve close games. Journal of Sports Science \& Medicine 9(1): 51-55. 